\title{
The predictive role of platelet to lymphocyte ratio in the occurrence of anastomotic complications following gastric resections for neoplasia - single centre experience
}

\author{
Călin Molnar ${ }^{1}$, Cosmin Lucian Nicolescu ${ }^{2 *}$, Marian Botoncea ${ }^{1}$, Vlad-Olimpiu \\ Butiurca $^{1}$, Bogdan Andrei Suciu ${ }^{2}$, Ioana Hălmaciu ${ }^{2}$, Liana Bianca Grigorescu ${ }^{3}$, \\ Septimiu Voidazan ${ }^{4}$ \\ 1. First Department of Surgery, George Emil Palade University of Medicine, Pharmacy, Science, and \\ Technology of Targu Mures, Romania \\ 2. Department of Anatomy and Embriology, George Emil Palade University of Medicine, Pharmacy, \\ Science, and Technology of Targu Mures, Romania \\ 3. Department of Physiopathology, George Emil Palade University of Medicine, Pharmacy, Science, \\ and Technology of Targu Mures, Romania \\ 4. Department of Epidemiology, George Emil Palade University of Medicine, Pharmacy, Science, and \\ Technology of Targu Mures, Romania
}

\begin{abstract}
Introduction: Our study investigated the importance of inflammation markers - ratio of platelets and lymphocytes (PLR), ratio of neutrophils and lymphocytes (NLR) and ratio of lymphocytes and monocytes (LMR) - as predictive markers in the occurrence of fistula or stenosis in patients diagnosed with gastric adenocarcinoma who underwent gastric resections. Materials and Methods: We conducted a retrospective study of 178 patients diagnosed with gastric adenocarcinoma. The included patients were divided into 3 groups: group 1 (77 patients, who underwent lower gastrectomy), group 2 (27 patients, who had upper polar gastrectomy otherwise known as proximal gastrectomy), group 3 (74 patients, who underwent total gastrectomy). Ratios of PLR, NLR, respectively LMR were calculated for all patients. Results: Out of 178 patients 52 (29.2\%) developed postoperative stenosis and 16 patients $(9.0 \%)$ had postoperative fistulae. The occurrence of anastomotic stenosis was associated with significantly higher preoperative platelet counts $(p=0.043)$ and PLR values $(p=0.023)$. ROC curve analysis indicated that the optimal PLR value for the prediction of gastric stenosis was 198.4 (AUC=0.609, sensitivity: 59.6\%, specificity: $61.9 \%$ ). For the prediction of fistulization PRL also displayed the highest performance among the analyzed hematological parameters (AUC $=0.561$, sensitivity: $43.7 \%$, specificity: $81.5 \%$, cut-off value 116.6 . Conclusion: Our study indicates the importance of PLR as e predictive factor in the occurrence of anastomotic complications (fistulae or stenosis) immediately following surgery in patients with gastric adenocarcinoma that undergo gastric resections. Further prospective studies on larger groups of patients are required, considering that PLR, NLR and LMR will be key markers in the clinical management of patients with gastric cancer.
\end{abstract}

*Corresponding author: Cosmin Lucian Nicolescu, Department of Anatomy and Embriology, George Emil Palade University of Medicine, Pharmacy, Science, and Technology of Targu Mures, Romania.

E-mail: cosminnicolescu@yahoo.com 
Keywords: gastric adenocarcinoma, neutrophil/lymphocyte ratio, lymphocyte/monocyte ratio, platelet/lymphocyte ratio

Received: $5^{\text {th }}$ December 2019; Accepted: $2^{\text {nd }}$ March 2020; Published: 10 ${ }^{\text {th }}$ March 2020

\section{Introduction}

Every year, approximately 1,040,000 people are diagnosed with gastric cancer worldwide. Around 782,000 die from the disease, making it the fifth most frequent and third most common cause of cancer death $(1,2)$.

The highest incidences are recorded in East Asia, Eastern Europe and South America, with the lowest rates observed in North America and most parts of Africa (3). According to Globocan sources in 2018 (4), in Romania there are 3,530 new cases per year (seventh place as frequency) and 3,020 deaths (fifth place as frequency).

The preferred treatment for advanced gastric cancer is surgery $(5,6)$. For some patients, without the chance of surgical treatment, the ultimate goal of complete treatment is to prolong survival and improve the quality of life. With improved surgical techniques and progress in traditional radiotherapy, chemotherapy and neoadjuvant therapy, the five-year survival rate of early gastric cancer can reach over $95 \%(7,8)$.

Inflammation is a hallmark of cancer, which contributes substantially to the development and progression of malignancy. In established gastric cancer, there is growing evidence for the roles that the local immune response and systemic inflammation play in tumour progression and patient survival (9). In recent years, more and more investigators have studied the importance of inflammation markers as prognostic factors in cancer pathogenesis $(9,10)$.

At present, the most important studied markers are the ratio of neutrophil level to lymphocyte level (NLR), the ratio of lymphocyte level to level of monocytes (LMR) and the ratio of platelets to lymphocyte level (PLR) (11-16).
Two of the main complications that increase postoperative morbidity and mortality in patients with gastric cancer following surgical resection are anastomosis fistula and anastomotic stenosis. Our study investigated NLR, LMR and PLR as predictive scores in patients with gastric cancer undergoing surgery (total and subtotal gastrectomies) and to evaluate their prognostic utility in correlation with the risk of complication such as anastomotic stenosis or fistula.

\section{Materials and Methods}

\section{Study participants}

The research was performed based on a retrospective observational study, for a period of six years (2014-2019), using the medical records of the General Surgery Clinic Section No. 1 within the Emergency County Clinical Hospital, Târgu Mureș, Romania, and it has the approval of the hospital's Ethics Committee (No. 31960/12.11.2019). We included 178 patients diagnosed with gastric cancer who were operated on in this clinic.

We included in the study patients diagnosed with gastric and a histopathological diagnosis of adenocarcinoma who underwent curative resection. We excluded from the study patients who underwent gastric resection for palliative purposes, as well as those where the histopathological examination of the gastric resection piece revealed tumour infiltration of the resection margins.

The data used in the study were obtained from the patients' general clinical observation sheets; we extracted demographic data (age and sex), main diagnosis, postoperative diagnosis, type of surgery, postoperative complications (suppuration, anastomotic stenosis, anastomotic fistulae). In all the patients studied, upon hospital admis- 
sion, one day prior to surgery, routine blood tests were collected (biochemistry: haematocrit, haemoglobin; complete blood cells count: neutrophils, lymphocytes, monocytes and platelets). Based on these, the value of the NLR, LMR and PLR levels was calculated in all patients.

Patients were divided into three groups according to the type of surgery: group 1 - inferior polar subtotal gastrectomy, group 2 - superior polar subtotal gastrectomy and group 3 - total gastrectomy.

Anastomotic fistula diagnosis was established based on the aspect of drain tube secretions or secondary to imaging studies (barium swallow). The diagnosis of anastomotic stenosis was established if during the postoperative period after the resumption of the oral feeding, the patients presented with vomiting, and on the esophageal gastric examination the existence of anastomotic stenosis was detected.

The diagnosis of anastomotic fistula was established based on intraperitoneal drainage tube secretions (intestinal secretions) or postoperative barium swallow test result. The diagnosis of anastomotic stenosis was established if during the postoperative period, after the resumption of oral intake, the patient presented vomiting and, on barium swallow test the existence of anastomotic stenosis was detected.

\section{Statistical analysis}

We used the SPSS statistical software package 20.0 (SPSS, Inc., Chicago, IL, USA) for all statistical analyses. The association between qualitative variables was assessed using the Chi-square test or the Fisher exact test. Quantitative data were presented as mean and standard deviation (for normally distributed data) and median/minmax (for abnormally distributed data). To check for differences between independent groups of quantitative data, the Mann Whitney test, Kruskal-Wallis test and ANOVA test were applied. Post-hoc analysis based on Dunn's approach with Bonferroni correction was also performed to identify the source of difference. The stenosis and fistulisation status was assessed against several explanatory variables. An ROC curve analysis was performed to evaluate discriminant accuracy and to find the cut-off values for the studied ratios (NLR, LMR and PLR). For all statistical tests, significance was set at $\mathrm{p} \leq 0.05$.

\section{Results}

The group consisted of 178 patients, aged 68.9 +/- 10.3 years (mean +/- SD), with a minimum of 29 years and a maximum of 91 years. Gender data analysis shows that $70.2 \%$ were males, with a distribution of $64.9 \%$ (group 1 - lower polar subtotal gastrectomy), $81.5 \%$ (group $2-$ upper polar subtotal gastrectomy) and 71.6\% (group 3 - total gastrectomy). The most postoperative deaths were recorded for group 2 (18.5\%), most fistulas for group $3(14.9 \%)$ and most cases of stenosis for group $1(39.0 \%)$. The differences for the three variables (death, fistula and stenosis) according to distribution by group were statistically significant (Chi-square test: $p$ $=0.002, \mathrm{p}=0.026, \mathrm{p}=0.042$ ). No statistically significant differences were identified between the three groups for the median values of neutrophils, lymphocytes or monocytes, or their ratios (NLR, LMR, PLR), respectively for the mean values of haemoglobin and platelet values, except for medium values of haematocrit ( $p$ $=0.045)$, (Table 1).

Table 2 shows the comparisons between median values of neutrophils, lymphocytes, monocytes and platelets. We applied the Mann-Whitney test between all patients with stenosis versus without stenosis, and between all patients with fistula versus without risk of fistulisation. We found a statistically significant increase in the case of platelet number of patients with stenosis $(p=0.043)$ and also for PLR $(p=0.023)$. 


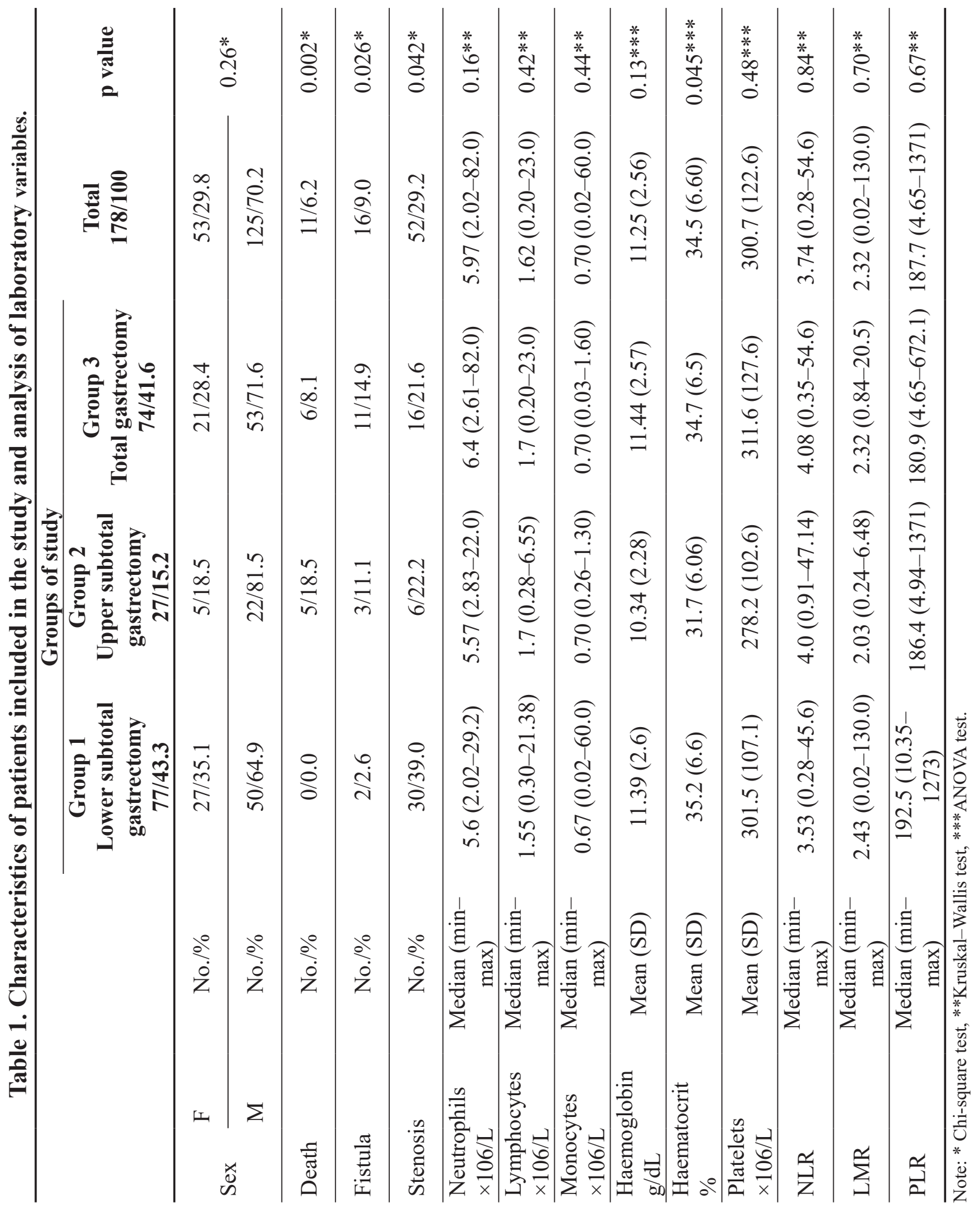


Table 2. Inferential comparisons between serum levels of variables of interest depending on the presence or absence of stenosis or fistula.

\begin{tabular}{lccc}
\hline & Stenosis Yes & Stenosis No & p value* \\
\hline Neutrophils, median (min-max) & $6.050(2.02-82.00)$ & $5.925(2.20-29.20)$ & 0.912 \\
\hline Lymphocytes, median (min-max) & $1.490(0.20-21.38)$ & $1.680(0.28-23.00)$ & 0.174 \\
\hline Monocyte, median (min-max) & $0.640(0.03-6.00)$ & $0.700(0.02-60.00)$ & 0.462 \\
\hline Platelets, median (min-max) & $319.5(22.00-592.00)$ & $282.0(9.60-844.00)$ & 0.043 \\
\hline NLR, median (min-max) & $4.585(0.28-54.67)$ & $3.654(0.35-47.14)$ & 0.314 \\
\hline LMR, median (min-max) & $2.318(0.16-23.76)$ & $2.364(0.02-130.00)$ & 0.832 \\
\hline PLR, median (min-max) & $223.0(10.35-1273)$ & $178.5(4.65-1371)$ & 0.023 \\
\hline & Fistula Yes & Fistula No & p value* \\
\hline Neutrophils, median (min-max) & $6.23(2.94-13.60)$ & $5.925(2.02-82.00)$ & 0.476 \\
\hline Lymphocytes, median (min-max) & $1.310(0.58-4.13)$ & $1.645(0.20-23.00)$ & 0.784 \\
\hline Monocyte, median (min-max) & $0.700(0.23-1.57)$ & $0.700(0.02-60.00)$ & 0.933 \\
\hline Platelets, median (min-max) & $247.5(9.60-445.00)$ & $291.5(22.00-844.00)$ & 0.132 \\
\hline NLR, median (min-max) & $4.358(0.71-17.00)$ & $3.709(0.28-54.67)$ & 0.686 \\
\hline LMR, median (min-max) & $2.000(0.84-17.96)$ & $2.400(0.02-130.00)$ & 0.604 \\
\hline PLR, median (min-max) & $167.5(4.948-767.20)$ & $190.8(4.65-1371)$ & 0.420 \\
\hline
\end{tabular}

*Note: Mann-Whitney test.

Using the ROC curves, the sensitivity and specificity performance parameters for risk of stenosis and postoperative fistulisation were determined depending on the cut-off level of the LMR, NLR and PLR ratios.

Comparative to the other ratios, PLR showed greater specificity and sensitivity both for the risk of stenosis $(\mathrm{AUC}=0.609 ;(95 \% \mathrm{CI}=0.533-$ $0.681)$, as well as for the risk of fistulae (AUC = 0.561 ; $(95 \% \mathrm{CI}=0.485-0.635))$.
The individual cut-offs, sensitivity and specificity parameters calculated for each report both for stenosis risk and fistulae risk are shown in Table 3.

Analysis of the ROC and the performance parameters was performed individually for each of the three groups, both for assessing the risk of stenosis and for assessing the risk of fistulisation.

Table 3. Cut-off values and performance parameters of LMR, NLR and PLR, for risk of stenosis and risk of fistulisation (for all group)

\begin{tabular}{lccccc}
\hline Reports & Cut-off level & Sensitivity & $\mathbf{9 5 \% C I}$ for sensitivity & Specificity & 95\%CI for specificity \\
\hline Stenosis & & & & & \\
\hline LMR & 3.03 & 73.1 & $59.0-84.4$ & 34.1 & $25.9-43.1$ \\
\hline NLR & 4.62 & 50.0 & $35.8-64.2$ & 62.7 & $53.6-71.1$ \\
\hline PLR & 198.4 & 59.6 & $45.1-73.0$ & 61.9 & $52.8-70.4$ \\
\hline Fistulisation & & & & & \\
\hline LMR & 2 & 56.2 & $29.9-80.2$ & 61.1 & $53.1-68.7$ \\
\hline NLR & 2.22 & 93.7 & $69.8-99.8$ & 22.2 & $16.1-29.4$ \\
\hline PLR & 116.6 & 43.7 & $19.8-70.1$ & 81.5 & $74.6-87.1$ \\
\hline
\end{tabular}




\section{For stenosis risk}

For all three subgroups, comparative to other ratios, PLR was more specific and sensitive: the lower polar subtotal gastrectomy group $(\mathrm{AUC}=$ $0.653,95 \% \mathrm{CI}=0.536-0.758)$, the superior polar subtotal gastrectomy group, $(\mathrm{AUC}=0.556,95 \%$ $\mathrm{CI}=0.353-0.745)$, and for the total gastrectomy group, $(\mathrm{AUC}=0.613,95 \% \mathrm{CI}=0.492-0.724)$, (Table 4).

\section{Assessment of the risk of fistulisation}

For the lower polar subtotal gastrectomy group, and for the total gastrectomy group the highest AUC was for PLR (AUC $=0.667 \%, 95 \% \mathrm{CI}=$ $0.550-0.770$ ), respectively (AUC $=0.593,95 \%$ $\mathrm{CI}=0.473-0.706)$.

For the superior polar subtotal gastrectomy group, the highest AUC was for NLR (AUC = $0.687,95 \% \mathrm{CI}=0.482-0.851)$, (Table 5).

\section{Discussion}

Inflammation is seen as the 'seventh attribute of cancer' and contributes to tumour proliferation, angiogenesis, metastasis, and resistance to chemotherapy and hormone therapy $(17,18)$. Various clinical and epidemiological studies have shown the link between chronic inflammation and gastric cancer (19).

In the literature, we have identified studies that independently investigated NLR and PLR with evaluation of the clinical significance of these markers in patients with various malignancies, including gastric cancer, both in terms of the oncological prognosis as well as in the immediate postoperative period $(11,13,17,20-24)$. Tests to determine the value of neutrophils, platelets and lymphocytes are routine and performed on all patients diagnosed with malignancies, and do not involve increased financial costs. Our study assessed NLR, PLR and LMR to predict tumour response and prognostic risk of complications in gastric cancer patients undergoing three types of intervention: inferior polar subtotal gastrectomy, superior polar subtotal gastrectomy and total gastrectomy. Where previous studies had analysed these markers in relation to disease progression or the presence of peritoneal metastases, we correlated them according to the risk of anastomotic complications in the postoperative period (stenotic risk or fistulae risk).

The appearance of an anastomosis fistula due to the associated infectious syndrome causes a systemic inflammatory syndrome. Also, the ap-

Table 4. Cut-off values and performance parameters of LMR, NLR and PLR, for risk of stenosis (for each of the three groups)

\begin{tabular}{lccccccc}
\hline Reports & AUC & $\begin{array}{c}\mathbf{9 5 \% C I} \text { for } \\
\text { AUC }\end{array}$ & $\begin{array}{c}\text { Cut-off } \\
\text { level }\end{array}$ & Sensitivity & $\begin{array}{c}\mathbf{9 5 \% C I} \text { for } \\
\text { sensitivity }\end{array}$ & Specificity & $\begin{array}{c}\mathbf{9 5 \% C I} \text { for } \\
\text { specificity }\end{array}$ \\
\hline Lower polar subtotal gastrectomy group & & & & & \\
\hline LMR & 0.531 & $0.414-0.646$ & 1.72 & 40.0 & $22.7-59.4$ & 74.5 & $59.7-86.1$ \\
\hline NLR & 0.554 & $0.436-0.667$ & 4.62 & 50.0 & $31.3-68.7$ & 68.1 & $52.9-80.9$ \\
\hline PLR & 0.653 & $0.536-0.758$ & 217 & 63.3 & $43.9-80.1$ & 70.2 & $55.1-82.7$ \\
\hline \multicolumn{2}{l}{ Superior polar subtotal gastrectomy group } & & & & \\
\hline LMR & 0.528 & $0.328-0.721$ & 2.03 & 66.6 & $22.3-95.7$ & 52.4 & $29.8-74.3$ \\
\hline NLR & 0.516 & $0.317-0.711$ & 2.70 & 83.3 & $35.9-99.6$ & 42.8 & $21.8-66.0$ \\
\hline PLR & 0.556 & $0.353-0.745$ & 287.1 & 83.8 & $54.1-100.0$ & 33.3 & $14.6-57.0$ \\
\hline Total gastrectomy group & & & & & \\
\hline LMR & 0.533 & $0.413-0.650$ & 1.75 & 87.5 & $61.7-98.4$ & 31.1 & $19.5-44.5$ \\
\hline NLR & 0.545 & $0.425-0.662$ & 4.16 & 56.2 & $29.9-80.2$ & 56.9 & $43.2-69.8$ \\
\hline PLR & 0.613 & $0.492-0.724$ & 120.1 & 93.8 & $79.4-100.0$ & 31.1 & $19.5-44.5$ \\
\hline
\end{tabular}


Table 5. Cut-off values and performance parameters of LMR, NLR and PLR, for risk of fistulisation (for each of the three groups)

\begin{tabular}{lccccccc}
\hline Reports & AUC & $\begin{array}{c}\mathbf{9 5 \% C I} \\
\text { for AUC }\end{array}$ & $\begin{array}{c}\text { Cut-off } \\
\text { level }\end{array}$ & Sensitivity & $\begin{array}{c}\mathbf{9 5 \% C I} \text { for } \\
\text { sensitivity }\end{array}$ & Specificity & $\begin{array}{c}\mathbf{9 5 \% C I} \text { for } \\
\text { specificity }\end{array}$ \\
\hline Lower polar subtotal gastrectomy group & & & & & & \\
\hline LMR & 0.540 & $0.423-0.654$ & 4.44 & 50.0 & $35.8-98.7$ & 82.6 & $72.2-90.4$ \\
\hline NLR & 0.527 & $0.410-0.642$ & 4.66 & 95.0 & $65.8-98.6$ & 38.6 & $27.6-50.6$ \\
\hline PLR & 0.667 & $0.550-0.770$ & 218.4 & 93.5 & $75.8-98.6$ & 42.6 & $31.3-54.6$ \\
\hline \multicolumn{2}{l}{ Superior polar subtotal gastrectomy group } & & & & \\
\hline LMR & 0.535 & $0.334-0.727$ & 2.12 & 66.6 & $49.4-99.2$ & 62.5 & $40.6-81.2$ \\
\hline NLR & 0.687 & $0.482-0.851$ & 9.16 & 66.6 & $49.4-99.2$ & 87.5 & $67.6-97.3$ \\
\hline PLR & 0.618 & $0.412-0.797$ & 374.3 & 66.6 & $49.4-99.2$ & 87.5 & $67.6-97.3$ \\
\hline Total gastrectomy group & & & & & \\
\hline LMR & 0.566 & $0.445-0.681$ & 2 & 63.6 & $30.8-89.1$ & 58.7 & $45.6-71.0$ \\
\hline NLR & 0.504 & $0.385-0.622$ & 2.9 & 45.5 & $16.7-76.6$ & 73.1 & $60.3-83.4$ \\
\hline PLR & 0.593 & $0.473-0.706$ & 116.6 & 45.5 & $16.7-76.6$ & 84.1 & $72.7-92.1$ \\
\hline
\end{tabular}

pearance of anastomosis stenosis after gastric resection frequently causes the expansion of the supra-anastomotic digestive tract due to stagnation of the secretions and can trigger a systemic inflammatory syndrome (25).

Initially, we analysed LMR, NLR and PLR for the whole group of patients (178 cases), evaluating their prognostic potential as strategic markers in managing patients with advanced gastric cancer with and without stenosis, and with and without risk of postoperative fistulisation. The median platelet count was significantly higher in patients with stenosis compared to those without stenosis $(\mathrm{p}=0.043)$, and therefore PLR was also statistically significantly higher $(p=0.023)$. In contrast, we did not identify statistically significant differences for the other markers, LMR and NLR, according to the presence or absence of stenosis and risk of fistulisation.

Using statistical software and based on ROC curves, we established the cut-off values of LMR, NLR and PLR that would be associated with the risk of anastomotic stenosis or fistulae. Between the three ratios, the most sensitive and specific was PLR, with an AUC of 0.609 (95\% CI $=0.533-0.681)$ and a cut-off level of $198.4(\mathrm{SE}=59.6 \%, 95 \% \mathrm{CI}=45.1-73.0 \%$; $\mathrm{SP}$
$=61.9 \%, 95 \% \mathrm{CI}=52.8-70.4 \%)$. At PLR values greater than 198.4 there was a high statistical probability that the anastomotic stenosis would occur, and at PLR values over 116.6 there was a sufficient statistical probability that the patient will have an anastomosis fistula as a postoperative complication.

We identified the most stenoses (30 patients out of 178) in the inferior polar subtotal gastrectomy group. Here the cut-off level for PLR was $217(\mathrm{SE}=63.3 \%, 95 \% \mathrm{CI}=43.9-80.1 \%$; SP $=70.2 \%, 95 \% \mathrm{CI}=55.1-82.7 \%)$. Lower polar gastrectomies (3/4 or $4 / 5$ with lymphadenectomy D1) left the possibility of gastro-duodenal end-to-end anastomosis (20 cases), end-to-side gastro-jejunal anastomoses to omega (50 cases), end-to-side gastro-jejunum on the Y loop in Roux (six cases), and terminal-terminal on the $Y$ loop in Roux (one case). In the case of superior polar gastrectomies, the stenosing character of the tumour leads only to the appearance of a supratumoural oesophageal dilatation that will be an anastomotic partner with consistency changes but also with tissue changes that may predispose to fistula (three cases). Although several stenoses were registered in the inferior polar subtotal gastrectomy group, the median PLR values 
between the three study groups were similar, without significant difference $(\mathrm{p}=0.67$; MannWhitney test). Despite this, PLR cut-off levels differ depending on the type of intervention, and therefore on the anatomical position of the gastric tumour. Of the total number of patients, 16 $(9 \%)$ had fistulas, most of them undergoing total gastrectomy. Here the cut-off level for PLR was $116.6(\mathrm{SE}=45.5 \%, 95 \% \mathrm{CI}=16.7-76.6 \%$; $\mathrm{SP}=$ $84.1 \%, 95 \% \mathrm{CI}=72.7-92.1 \%)$.

Hirahara's study (11) demonstrated a close association between adverse gastric cancer progression and high PLR $(>248.4)$ or NLR $(>2.46)$. Wang et al. (17) evaluated 120 patients with unresectable gastric cancer and reported that patients with high baseline PLR or NLR had a significantly lower response to chemotherapy. PLR is a potential prognostic factor for cancers associated with high thrombotic risk, such as pancreatic cancer, so the thrombotic profile of basic cancer should be followed (24). The use of PLR as a prognostic factor may not be accurate with respect to relatively hypocoagulative cancers, such as breast cancer (26), but as gastric cancer is hypercoagulative (24), baseline levels of PLR and NLR may provide valuable prognostic information.

Our study has some limitations. First, it is a preliminary study based on retrospective analysis of a small group $(\mathrm{n}=175)$ from a single institution. This retrospective collection did not allow the identification of data (number of deaths, time of death) in order to perform a survival analysis of gastric cancer patients according to the cut-off levels of the studied ratios.

However, there was also the possibility that the patients studied were very heterogeneous, with comorbidities that could influence the value of analysed ratios. On the other hand, increased levels of PLR and NLR play a central role in a systemic inflammation not only in oncologic pathologies such as gastric, oesophageal, hepa- tocellular, pancreatic and colorectal cancer, but also in other diseases like acute kidney injury (27).

These limitations could have led to bias influencing the results of the study. Therefore, larger validation studies are needed to confirm our findings, in the context of routine, inexpensive tests. These may also be of clinical utility for patients with other malignancies, such as oesophageal, hepatocellular, pancreatic and colorectal cancer.

\section{Conclusion}

Our study showed the importance of PLR as a predictive factor for the appearance of fistula, respectively of anastomotic stenosis in the immediate postoperative period in patients with gastric adenocarcinomas that underwent gastric resections. Further prospective studies on larger groups of patients are required, considering that PLR, NLR and LMR will be key markers in the clinical management of patients with gastric cancer.
Abbreviations
AUC: area under curve
CI: confidence interval
LMR: lymphocyte to monocyte ratio
NLR: neutrophil to lymphocyte ratio
PLR: platelet to lymphocyte ratio
ROC: Reciever Operating Characteristic
SE: sensitivity
SP: specificity

\section{Authors' Contributions}

CM: Conceptualization, methodology, writing original draft

CLN: Conceptualization, supervision;

MB: Investigation, writing - review \& editing;

OVB: Data curation, formal analysis;

BAS: Conceptualization, methodology;

IH: Methodology, writing - review \& editing; 
BLG: Methodology, supervision;

SV: Data curation, investigation, methodology.

\section{Conflicts of Interest}

The authors declare no conflict of interest.

\section{References}

1. Ferlay J, Colombet M, Soerjomataram I, Mathers C, Parkin D.M, Pineros M, et al. Estimating the global cancer incidence and mortality in 2018: GLOBOCAN sources and methods. Int J Cancer. 2018;144(8):194153. DOI: $10.1002 /$ ijc. 31937

2. deMartel C, Parsonnet J. Stomach cancer. In: MJ Thun, MS Linet, JR Cerhan, CA Haiman, D, Schottenfeld eds. Cancer epidemiology and prevention. 4th ed. New York: Oxford University Press; 2018:593-610.

3. Freddie B, Ferlay J, Soerjomataram I, Siegel R, Torre L.A, Jemal A. Global Cancer Statistics 2018: GLOBOCAN Estimates of Incidence and Mortality Worldwide for 36 Cancers in 185 Countries. Cancer J Clin. 2018;68:394-424 DOI: 10.3322/caac.21492

4. https://gco.iarc.fr/today/data/factsheets/populations/642-Romania-fact-sheets.pdf

5. He W, Tu J, Huo Z. Surgical interventions for gastric cancer: a review of systematic reviews. Int J Clin Exp Med. 2015;8:13657-13669.

6. Tang HN, Hu JH. A comparison of surgical procedures and postoperative cares for minimally invasive laparoscopic gastrectomy and open gastrectomy in gastric cancer. Int J Clin Exp Med. 2015;8:10321-10329.

7. Song $\mathrm{Z}, \mathrm{Wu} \mathrm{Y}$, Yang J, Yang D, Fang X. Progress in the treatment of advanced gastric cancer. Tumor Biol. 2017;39(7). DOI: 10.1177/1010428317714626

8. Karimi P, Islami F, Anandasabapathy S, Freedman ND, Kamangar F. Gastric cancer: descriptive epidemiology, risk factors, screening, and prevention. Cancer Epidemiol Biomarkers Prev. 2014;23(5):700-713. DOI: 10.1158/1055-9965.EPI-13-1057

9. Diakos CI, Charles KA, McMillan DC, Clarke SJ. Cancer-related inflammation and treatment effectiveness. Lancet Oncol. 2014:15(11):e493-503 DOI: 10.1016/ S1470-2045(14)70263-3

10. Roxburgh CS, McMillan DC. Cancer and systemic inflammation: treat the tumour and treat the host. Br J Cancer. 2014;110:1409-1412. DOI: 10.1038/ bjc. 2014.90

11. Hirahara T, Arigami T, Yanagita S, et al. Combined neutrophil-lymphocyte ratio and platelet-lymphocyte ratio predicts chemotherapy response and prognosis in patients with advanced gastric cancer. BMC Cancer. 2019;19(1):672. DOI: 10.1186/s12885-019-5903-y

12. Templeton AJ, Ace O, McNamara MG, Al-Mubarak M,
Vera-Badillo FE, Hermanns T, et al. Prognostic role of platelet to lymphocyte ratio in solid tumors: a systematic review and meta-analysis. Cancer Epidemiol Biomarkers Prev. 2014;23:1204-1212. DOI: 10.1158/10559965.EPI-14-0146

13. Yodying H, Matsuda A, Miyashita M, Matsumoto S, Sakurazawa N, Yamada M, et al. Prognostic Significance of Neutrophil-to-Lymphocyte Ratio and Platelet-to-Lymphocyte Ratio in Oncologic Outcomes of Esophageal Cancer: A Systematic Review and Meta-analysis. Ann Surg Oncol. 2016;23:646-654. DOI: 10.1245/s10434-015-4869-5

14. Zhao QT, Yuan Z, Zhang H, Zhang XP, Wang HE, Wang $\mathrm{ZK}$, et al. Prognostic role of platelet to lymphocyte ratio in non-small cell lung cancers: A meta-analysis including 3,720 patients. Int J Cancer. 2016;139:164-170. DOI: 10.1002/ijc.30060

15. Lu C, Gao P, Yang Y, Chen X, Wang L, Yu D, et al. Prognostic evaluation of platelet to lymphocyte ratio in patients with colorectal cancer. Oncotarget. 2017;8:86287-86295. DOI: 10.18632/oncotarget.21141

16. Mártha $\mathrm{O}$, Balan D, Porav-Hodade D, Drágus E, Vartolomei MD, Chibelean $\mathrm{CB}$, et al. The role of neutrophil to lymphocyte ratio in patients with pTa non-muscle invasive bladder cancer. Rev Romana Med Lab. 2020;28(1):29-38. DOI: 10.2478/rrlm-2020-0001

17. Wang F, Liu ZY, Xia YY, et al. Changes in neutrophil/ lymphocyte and platelet/lymphocyte ratios after chemotherapy correlate with chemotherapy response and prediction of prognosis in patients with unresectable gastric cancer. Oncol Lett. 2015;10(6):3411-3418. DOI: $10.3892 / \mathrm{ol} .2015 .3783$

18. Hussain SP, Harris CC. Inflammation and cancer: an ancient link with novel potentials. Int J Cancer. 2007;121:2373-2380. DOI: 10.1002/ijc.23173

19. Lee S, Oh SY, Kim SH, Lee JH, Kim MC, Kim KH, et al. Prognostic significance of neutrophil lymphocyte ratio and platelet lymphocyte ratio in advanced gastric cancer patients treated with FOLFOX chemotherapy. BMC Cancer. 2013;13:350. DOI: 10.1186/1471-240713-350

20. Nakamura N, Kinami S, Fujii Y, et al. The neutrophil/ lymphocyte ratio as a predictor of peritoneal metastasis during staging laparoscopy for advanced gastric cancer: a retrospective cohort analysis. World J Surg Oncol. 2019;17(1):108. DOI: 10.1186/s12957-019-1651-3

21. Koh CH, Bhoo-Pathy N, Ng KL, Jabir RS, Tan GH, See $\mathrm{MH}$, et al. Utility of pre-treatment neutrophil-lymphocyte ratio and platelet-lymphocyte ratio as prognostic factors in breast cancer. Br J Cancer. 2015;113:150158. DOI: $10.1038 /$ bjc. 2015.183

22. Chan JC, Chan DL, Diakos CI, Engel A, Pavlakis N, Gill A, et al. The Lymphocyte-to-Monocyte Ratio is a Superior Predictor of Overall Survival in Comparison to Established Biomarkers of Resectable Colorectal 
Cancer. Ann Surg. 2017;265:539-546. DOI: 10.1097/ SLA.0000000000001743

23. Sierzega M, Lenart M, Rutkowska M, Surman M, My$\operatorname{tar}$ B, Matyja A, et al. Preoperative neutrophil-lymphocyte and lymphocyte-monocyte ratios reflect immune cell population rearrangement in Resectable pancreatic Cancer. Ann Surg Oncol. 2017;24:808-815. DOI: 10.1245/s10434-016-5634-0

24. Heras P, Hatzopoulos A, Kritikos N, Kritikos K. Platelet count and tumor progression in gastric cancer patients. Scand J Gastroenterol. 2010;45:1005-1006. DOI: $10.3109 / 00365521003797221$

25. Dobru D, Boeriu A, Mocan S, Pascarenco O, Boeriu
C, Molnar C. Gastric carcinoid and therapeutic options, Case report and review of the literature. J Gastrointestin Liver Dis. 2013;22(1):93-6.

26. Seretis C, Seretis F, Lagoudianakis E, Politou M, Gemenetzis G, Salemis NS. Enhancing the accuracy of platelet to lymphocyte ratio after adjustment for large platelet count: a pilot study in breast cancer patients. Int J Surg Oncol. 2012;2012:653608. DOI: $10.1155 / 2012 / 653608$

27. Mertoglu C, Gunay M, Gurel A, Gungor M, Gul V. Ischemic modified albumin increases in acute kidney injury. Rev Romana Med Lab. 2018;26(1):37-43. DOI: 10.2478/rrlm-2018-0002 